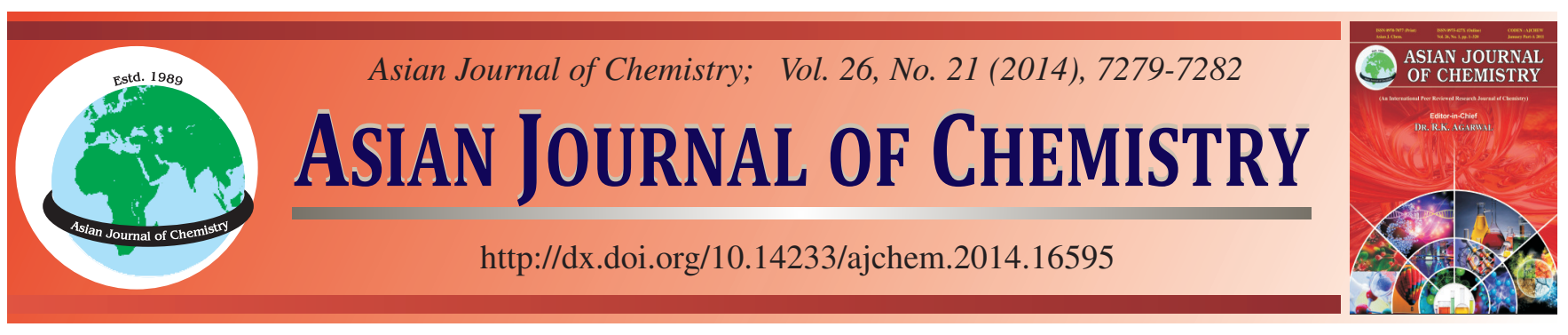

\title{
Analysis of Cypermethrin and Permethrin in Celery Cabbage by Lanthanum-Modified Activated Alumina QuEChERS Method
}

\author{
FengXia Qiao*, Zhiping Lin and MengGe Wang
}

${ }^{1}$ Department of Biochemistry, Baoding University, Qiyi Dong Road 3027, Baoding, P.R. China

*Corresponding author: Tel: +86 312 5972186, E-mail: qiaofengxia@126.com

\begin{abstract}
A new QuEChERS method based on lanthanum-modified activated alumina for the determination of cypermethrin and permethrin in celery cabbage was proposed. The lanthanum-modified activated alumina that prepared by glycerol complexing-assisted sol gel method using lanthanum as the modifier was employed as the adsorbent of QuEChERS for purification of cypermethrin and permethrin residues in celery cabbage coupled with gas chromatography detection and ethyl acetate as extraction solvent. Factors that affected QuEChERS efficiency were investigated including extraction solvent, extraction time, adsorbent kinds and adsorption time etc. The results showed that this lanthanum-modified activated alumina adsorbent could effectively purify the sample and eliminate the interferences originated from sample matrices. Under the optimized conditions, the fortified recoveries at three spiked levels were ranged from 91.6 to $110 \%$ with $\mathrm{RSD} \leq 4.9 \%$. Good linearity of the two pesticides was ranged from 0.005 to $1.190 \mathrm{mg} / \mathrm{g}$ with $\mathrm{r} \geq 0.9994$.
\end{abstract}

Keywords: QuEChERS; Permethrin, Cypermethrin, Activated alumina.

\section{INTRODUCTION}

Cypermethrin and permethrin are two kinds of pyrethroid pesticides used in agriculture to control insects. They are among the most toxic substances that contaminating food, environment, fruits and vegetables ${ }^{1}$. Therefore, it is crucial to monitor cypermethrin and permethrin residues in fruits and vegetables.

At present, the determination of pesticide residues in complicated matrices was a formidable challenge mainly because of the trace level of analytes and a large amount of interferences, so the sample must initially be cleaned up before final analysis ${ }^{2-5}$. Generally, solvent extraction ${ }^{6}$, matrix solidphase dispersion extraction ${ }^{7}$, gel-permeation chromatography ${ }^{8}$, emulsification-mixroextraction $^{9,10}$, solid-phase extraction ${ }^{11,12}$ and liquid-phase microextraction ${ }^{13}$ etc. were often employed for sample extraction and purification. However, from the practical point of view, these pretreatment procedures suffered from non-equilibrium extraction procedures or the small contact surface between the phases, which negatively affected the sample throughput and the enrichment factors.

QuEChERS (quick, easy, cheap, effective, rugged and safe) technique as a novel sample extraction and clean-up method was developed for analysis of multiple residues in agricultural products $^{14}$. It was a streamlined and effective extraction and cleanup approach for analysis of various analytes in food matrices ${ }^{15,16}$. The QuEChERS procedures had the characters of few simple analytical steps, fast and easy to perform and less susceptible to errors ${ }^{17}$ and it could provide high recovery for a broad scope of pesticides ${ }^{18}$. In QuEChERS, extraction solvent and purification adsorbent were the main key factors that affected purification efficiency. The original extraction solvent of QuEChERS was acetonitrile with primary-secondary amine (PSA) as the adsorbent. For the higher toxicity of acetonitrile and the primary-secondary amine adsorbent only be good at removing of fatty acid, not suitable for sterol, pigment and vitamin elimination. Many modification/improvement work of the QuEChERS was proposed to ensure efficient extraction of the analytes, minimize the toxic of the extraction system or expand the spectrum of matrices covered. Of which, ethyl acetate, dry ice or acetate-acetonitrile etc as extraction solvent and graphite carbon, $\mathrm{C}_{18}$ or neutral alumina oxide etc. as adsorbent had been developed ${ }^{19,20} . \mathrm{C}_{18}$ and graphite carbon black was suitable for adsorption of sterol, pigment and vitamin. While neutral alumina oxide could eliminate part water solubility pigment and most of lipide pigment ${ }^{7}$. Though these measurement made a great improvement. The QuEChERS method still suffered from too complex samples analysis, new extraction system of QuEChERS was desired greatly.

The purpose of this work was to develop a new QuEChERS method for determination of cypermethrin and permethrin in celery cabbage sample coupled with GC-FID detection. In the 
QuEChERS procedures, low toxicity solvent of ethyl acetate was employed as the extraction solvent with lanthanummodified activated alumina as the adsorbent. The lanthanummodified activated alumina adsorbent could effectively purify the sample and eliminate the sample matrices interferences. The proposed QuEChERS-GC method was suitable for extraction of cypermethrin and permethrin from complex vegetable samples.

\section{EXPERIMENTAL}

Cypermethrin and permethrin were obtained from Yangnong Chemical Co. Ltd. (Yangzhou, China). Ethyl acetate, aluminum sulfate, glycerol and lanthanum nitrate were obtained from Tianjin Jin Feng Chemical Co., Ltd. Dichloromethane, anhydrous $\mathrm{MgSO}_{4}$ and acetonitrile were of analytical grades and purchased from Kermel Chemical Co. Ltd. (Tianjin, China). Neutral alumina oxide and basic alumina oxide (100200 mesh) (obtained from National Pharmaceutical Group Chemical Reagent Co., Ltd.) were dried at $130^{\circ} \mathrm{C}$ for $12 \mathrm{~h}$ before use. All the other reagents used in the experiment were of the highest grade commercially available. Double deionized water was filtered with $0.45 \mu \mathrm{m}$ filter membrane before use.

Preparation of lanthanum-modified activated alumina: Aluminum sulfate solution $(200 \mathrm{~mL}, 1.28 \mathrm{~g} / \mathrm{mL})$ was adjusted to $\mathrm{pH} 7.5$ by aqueous ammonium hydroxide $(20 \%)$ under vigorous stirring. After stirring for $2 \mathrm{~h}$, the obtained $\mathrm{Al}(\mathrm{OH})_{3}$ was filtered and washed until none $\mathrm{SO}_{4}{ }^{2-}$ existed, then it was adjusted to $\mathrm{pH} 4$ by $0.1 \%$ aqueous $\mathrm{HNO}_{3}$ to obtain aluminium hydrogel. And then $306 \mathrm{~g}$ of glycerol and $2.7 \mathrm{~g}$ of lanthanum nitrate were added into the aluminium hydrogel and mixed sufficiently. The mixture was roasted at $540{ }^{\circ} \mathrm{C}$ for $10 \mathrm{~h}$ to obtain lanthanum-modified activated alumina.

Instrumentation and conditions: Cypermethrin and permethrin were analyzed on a Shimadzu GC-2014 system equipped with a FID detector (Shimadzu, Japan) and DB-5 column (5\% phenyl-methylpolysiloxane $30 \mathrm{~m} \times 0.53 \mathrm{~mm} \times 1$ $\mu \mathrm{m})$. High-purity nitrogen $(99.999 \%)$ was used as the carrier gas with flow rate at $5 \mathrm{~mL} / \mathrm{min}$ and split ratio of $2: 1$. A GH300 high-purity hydrogen generator and GA-2000A air pump (Beijing ZXHL Technology Development Co. Ltd.) were used to supply hydrogen and air at the rate of $30 \mathrm{~mL} / \mathrm{min}$ and 400 $\mathrm{mL} / \mathrm{min}$, respectively. The temperature-programmed mode was as follows: the initial oven temperature was set at $250{ }^{\circ} \mathrm{C}$ for 2 min, then ascended to $280{ }^{\circ} \mathrm{C}$ at the rate of $6{ }^{\circ} \mathrm{C} / \mathrm{min}$ and held for $12 \mathrm{~min}$. The injection port and detector temperatures were maintained at $285^{\circ} \mathrm{C}$ and $290{ }^{\circ} \mathrm{C}$, respectively.

Sample QuEChERS extraction: Different celery cabbage samples (80-100 g) were collected from the local markets and homogenized, then $3 \mathrm{~g}$ of the homogenized sample was mixed with the extraction solution (the mixture of $3 \mathrm{~mL}$ ethyl acetate and $0.5 \mathrm{~g} \mathrm{NaCl}$ ) and ultrasonicated for $2 \mathrm{~min}$. Then the mixture was centrifuged at $4000 \mathrm{rpm}$ for $5 \mathrm{~min}$ and the obtained supernatant $(1 \mathrm{~mL})$ was mixed with $0.2 \mathrm{~g}$ anhydrous $\mathrm{MgSO}_{4}$ and $0.1 \mathrm{~g}$ lanthanum-modified activated alumina. It was ultrasonicated for $2 \mathrm{~min}$, placidly placed for $5 \mathrm{~min}$ and centrifuged (4000 rpm) for $5 \mathrm{~min}$. The obtained supernatant was filtered through a $0.45 \mu \mathrm{m}$ membrane and employed $(1 \mu \mathrm{L})$ for further analysis.

\section{RESULTS AND DISCUSSION}

Selection of extraction solvent: The selection of extraction solvent was critical to obtain an economic recovery of the target analytes from complex sample matrix. Generally, acetonitrile was the recommended solvent for QuEChERS and with the help of salt would be separated more easily from water. Ethyl acetate had the character of partial miscibility with water but not suitable for large lipids and waxes samples, for it would co-extracts with them leading lower recoveries and bad purification efficiency. Considered the characrers of celery cabbage matrix, different extraction solvents including toluene, ethyl acetate, acetonitrile, $n$-hexane, acetonitrile-acetic acid (99:1, $\mathrm{v} / \mathrm{v})$, acetonitrile-toluene $(1: 1, \mathrm{v} / \mathrm{v})$ and acetonitrile-ethyl acetate $(1: 1, \mathrm{v} / \mathrm{v})$ were investigated to obtain the optimum extraction efficiency. Fig. 1 showed that acetonitrile and ethyl acetate had better ability for extraction cypermethrin and permethrin from celery cabbage. Considered the higher toxicity of acetonitrile, ethyl acetate was selected as the extraction solvent. Moreover, the effect of its volume on the extraction ratio of cypermethrin and permethrin was also investigated. Fig. 2 indicated that $3 \mathrm{~mL}$

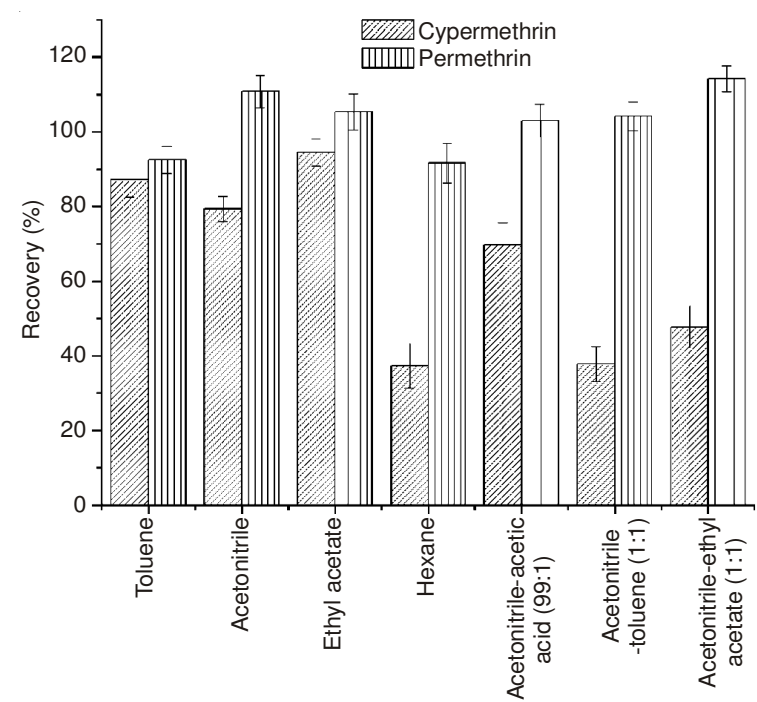

Fig. 1. Recovery obtained by different extraction solvent

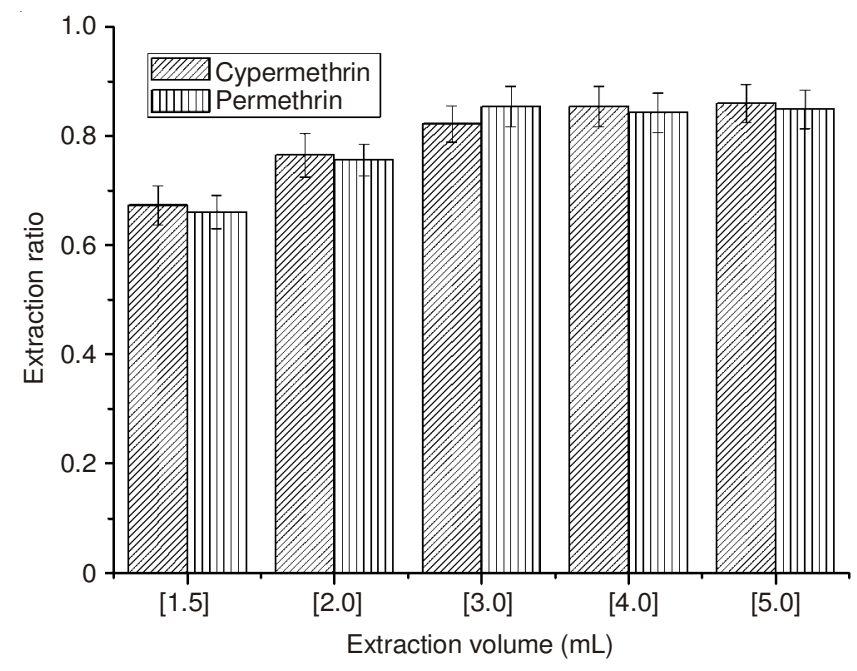

Fig. 2. Extraction ratio of different ethyl acetate volumes 
ethyl acetate could provide the satisfactory recovery. If the volume of ethyl acetate was less than $3 \mathrm{~mL}$, recoveries of the two analytes were increased with the increasing volume. While the volume of ethyl acetate was more than $3 \mathrm{~mL}$, the extraction ratio was kept stable. Compromised the sensitivity and the amount of organic solvent, $3 \mathrm{~mL}$ ethyl acetate was used for further work and $0.5 \mathrm{~g} \mathrm{NaCl}$ was added to induce phase separation.

In some instances, the $\mathrm{pH}$ of the extraction solvent must be controlled. For most of the pesticides, they are more stable at lower $\mathrm{pH}$. In this work, $\mathrm{pH}$ of extraction solvent ranged from 1 to 11 was investigated and the results indicated that $\mathrm{pH}$ ranging from 1 to 8 had none obvious influence on the extraction efficiency of cypermethrin and permethrin. When the $\mathrm{pH}$ was more than 8 , the extraction efficiency of cypermethrin and permethrin was decreased greatly with $\mathrm{pH}$ increased for ethyl acetate, cypermethrin and permethrin be decomposed. Due to the acidity of ethyl acetate being about 5 , none $\mathrm{pH}$ adjustment was employed in extraction procedure.

Extraction time of extraction solvent: The interaction time between extraction solvent and sample was one of factors that affected extraction efficiency. Different extraction time was investigated and the results (Fig. 3) indicated that the mass transfer between extraction solvent (ethyl acetate) and the homogenized celery cabbage was very fast. It could reach equilibrium within $2 \mathrm{~min}$. So extraction time of 2 min was employed for further work.

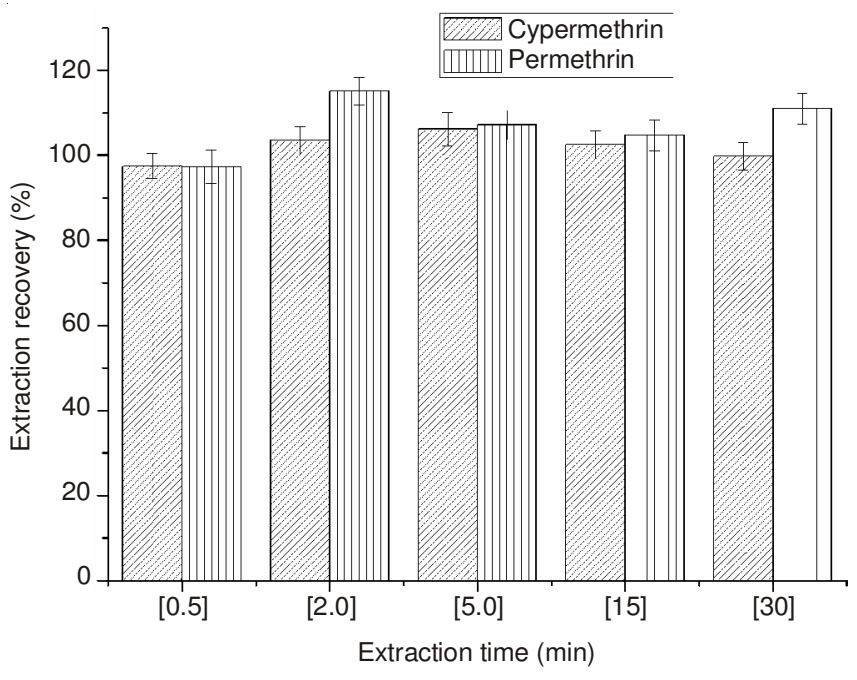

Fig. 3. Effect of extraction time on cypermethrin and permethrin recovery

Selection of adsorbent: The adsorbent was selected to retain matrix components, so a suitable adsorbent was a key factor for elimination of sample matrix impurities. An appropriate adsorbent and its amount should accord to the properties of matrix and the target compounds. To obtain the economic adsorbent, neutral alumina oxide, basic alumina oxide, diatomite, graphite carbon, lanthanum-modified activated alumina, activated alumina (it was prepared and treated in an identical manner as lanthanum-modified activated alumina, in absence of lanthanum nitrate), neutral alumina oxide-diatomite (1:1, $\mathrm{g} / \mathrm{g})$ and graphite carbon-diatomite $(1: 1, \mathrm{~g} / \mathrm{g})$ were taken into consideration except the original primary-secondary amine, which was considered expensive. Compared with the blank (none adsorbent), diatomite adsorbent brought new interferences into sample, the purification and recovery of basic alumina oxide (62.6-71.5\%) was not satisfied. Graphite carbon (52.4-61.8\%) and activated alumina (59.4-60.7\%) had better clean-up efficiency, but both of them had strong adsorption to cypermethrin and permethrin, leading to a decreased recovery. Though lanthanum-modified activated alumina and neutral alumina oxide adsorbent had the least interference peaks, the lanthanum-modified activated alumina gave better recovery (91.6-110\%) than that of neutral alumina oxide (84.5-92.3\%). Compromised the clean-up efficiency and recovery, lanthanummodified activated alumina was selected as the adsorbent of QuEChERS. Its amount (0.1-0.6 g) was investigated also and the results showed that the amount of lanthanum-modified activated alumina ranging from 0.1 to $0.4 \mathrm{~g}$ had not obviously influence on the two pesticides recoveries. However, when the amount was more than $0.4 \mathrm{~g}$, the recovery of the two analytes would be gradually decreased. Compromised the recovery and the purification ability, $0.1 \mathrm{~g}$ lanthanum-modified activated alumina was used as the optimized amount. Additionally, $0.2 \mathrm{~g}$ anhydrous $\mathrm{MgSO}_{4}$ was added into the sample simultaneously to remove much of the excess water, improve analytes partitioning and provide better clean up.

Adsorptive time of adsorbent: Adsorptive time (the time between the adsorbent and sample) must be suitable. Too short time, would make the impurities can't be completely adsorbed, while too long time, would lead to the impurities of adsorbent be dissolved out. After optimized, $5 \mathrm{~min}$ was employed as the compromised adsorbent time for sample impurities adsorption.

Method performance: The proposed QuEChERS-GC method was evaluated by linearity, precision, recovery, detection limits, inter-assay and intra-assay deviation. Under the optimum conditions, good linearities were ranged from 0.005 to $1.190 \mathrm{mg} / \mathrm{g}$ with linearity equation $\mathrm{y}=2.99 \times 10^{6} \mathrm{X}+$ 28799.51 and $y=4.13 \times 10^{6} \mathrm{X}+37928.16$ (where $\mathrm{Y}$ was the peak area, $X$ was the concentration of sample $(\mathrm{mg} / \mathrm{g})$ ) for cypermethrin and permethrin, respectively $(\mathrm{r} \geq 0.9994)$. The detection limits based on $\mathrm{S} / \mathrm{N}=3$ were $0.39 \mu \mathrm{g} / \mathrm{g}$ and 0.43 $\mu \mathrm{g} / \mathrm{g}$ for cypermethrin and permethrin, respectively. Intra-assay and inter-assay precision were expressed by the relative standard deviation (RSD), five replicates of the control samples at three spiked levels in the same day and three different days were analyzed with results less than 5.6 and $7 \%$, respectively. Additionally, chromatogram (Fig. 4) of spiked sample indicated that QuEChERS-GC exhibited more clean eluents without interferences, which demonstrated the high selectivity of the proposed QuEChERS-GC method.

Sample analysis: To illustrate a typical application of the developed QuEChERS-GC method, five kinds of celery cabbages that obtained from the local grocery stores were used for QuEChERS-GC analysis. None cypermethrin or permethrin was observed in any of the samples, which demonstrated that the misapplication of the two kinds of pesticide in celery cabbage at local area was not extensive. The recoveries of the two pesticides at three fortified levels indicated that the recoveries of cypermethrin and permethrin were ranged from 91.6 to $110 \%$ with RSD $\leq 4.9 \%$ (Table- 1 ). The chromatogram (Fig. 4) of the extract revealed that after the QuEChERS protocol, the samples were clean enough for direct GC analysis 
TABLE-1

RESULTS OF CELERY CABBAGE SAMPLES $(\mathrm{n}=5)$

\begin{tabular}{|c|c|c|c|c|c|}
\hline Analytes & Sample content $(\mathrm{mg} / \mathrm{g})$ & Spiked levels $(\mathrm{mg} / \mathrm{g})$ & Detected content $(\mathrm{mg} / \mathrm{g})$ & Recovery $(\%)$ & $\operatorname{RSD}(\%)$ \\
\hline \multirow{3}{*}{ Cypermethrin } & \multirow{3}{*}{$\mathrm{Nd}$} & 0.010 & 0.011 & 110.0 & 2.8 \\
\hline & & 0.050 & 0.050 & 100.1 & 4.9 \\
\hline & & 0.149 & 0.162 & 108.4 & 2.4 \\
\hline \multirow{3}{*}{ Permethrin } & \multirow{3}{*}{$\mathrm{Nd}$} & 0.010 & 0.009 & 91.6 & 3.9 \\
\hline & & 0.050 & 0.049 & 98.0 & 3.1 \\
\hline & & 0.149 & 0.163 & 109.6 & 2.5 \\
\hline
\end{tabular}
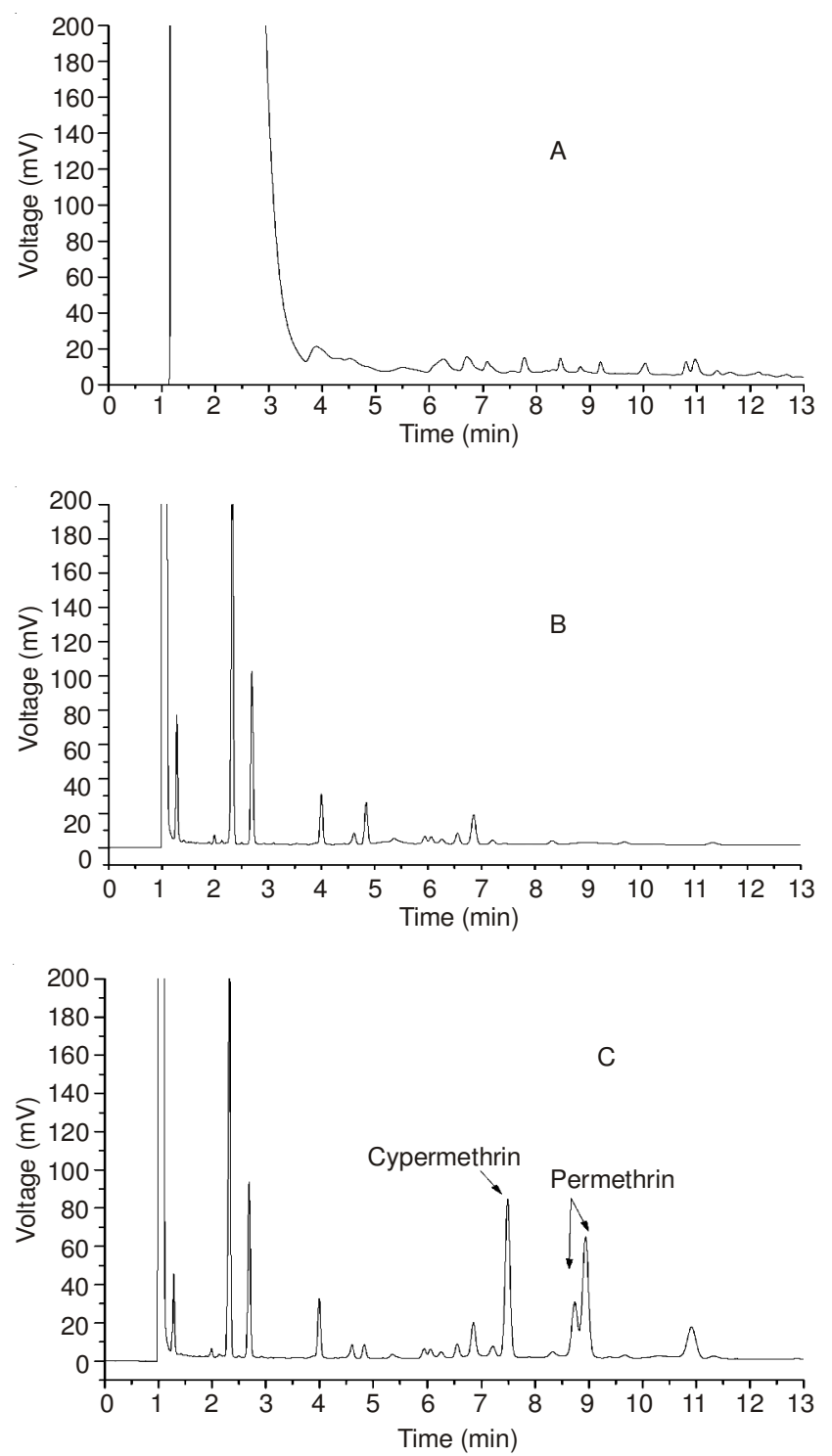

Fig. 4. Chromatogram of celery cabbage samples, (A: Unpurified sample B: Purified sample C: Fortified sample)

and no interferences originating from the human celery cabbage matrixes were observed. All this indicated that the method was reliable and could be used for the determination of trace cypermethrin or permethrin in celery cabbages.

\section{Conclusion}

A simple, low-cost and low toxicity QuEChERS-GC method for the determination of cypermethrin and permethrin pesticides in vegetables was developed. The method involved extraction with ethyl acetate, liquid-liquid partition with addition of $\mathrm{NaCl}$ followed by dispersive solid phase cleanup with lanthanum-modified activated alumina adsorbent and anhydrous $\mathrm{MgSO}_{4}$. After the QuEChERS protocol, the sample matrix interferences could be efficiently eliminated and the obtained sample was clean enough for direct GC analysis. The developed procedure gave satisfactory recovery (91.6-110\%) with RSD less than $4.9 \%$ for the studied pesticides in celery cabbage. It was suitable for analysis of permethrin and cypermethrin residues in celery cabbages.

\section{ACKNOWLEDGEMENTS}

The project sponsored by National Natural Science Foundation of China (31301464), Natural Science Foundation of Hebei province (B2012201052, B2011104002).

\section{REFERENCES}

1. J. Fenik, M. Tankiewicz and M. Biziuk, Trends Anal. Chem., 30, 814 (2011).

2. H. Yan, Y. Han and J. Du, Anal. Methods, 4, 3002 (2012).

3. A. Wilkowska and M. Biziuk, Food Chem., 125, 803 (2011).

4. H. Wang, H. Yan and J. Qiao, J. Sep. Sci., 35, 292 (2012).

5. B. Gilbert-López, J.F. García-Reyes and A. Molina-Díaz, Talanta, 79, 109 (2009).

6. H. Liu and P.K. Dasgupta, Anal. Chem., 68, 1817 (1996).

7. J. Cheng, M. Liu, Y. Yu, X. Wang, H. Zhang, L. Ding and H. Jin, Meat Sci., 82, 407 (2009).

8. E. Jover and J. Maria Bayona, J. Chromatogr. A, 950, 213 (2002).

9. C. Jia, X. Zhu, L. Chen, M. He, P. Yu and E. Zhao, J. Sep. Sci., 33, 244 (2010).

10. J. Regueiro, M. Llompart, C. Garcia-Jares, J.C. Garcia-Monteagudo and R. Cela, J. Chromatogr. A, 1190, 27 (2008).

11. Q. Zhou, Y. Ding, J. Xiao, G. Liu and X. Guo, J. Chromatogr. A, 1147, 10 (2007).

12. D.j. Djozan and Y. Assadi, Chromatographia, 60, 313 (2004).

13. C. Basheer, R. Balasubramanian and H.K. Lee, J. Chromatogr. A, 1016, 11 (2003).

14. T.D. Nguyen, J.E. Yu, D.M. Lee and G.-H. Lee, Food Chem., 110, 207 (2008).

15. A. Wilkowska and M. Biziuk, Food Chem., 125, 803 (2011).

16. C. Lesueur, P. Knittl, M. Gartner, A. Mentler and M. Fuerhacker, Food Contr., 19, 906 (2008).

17. S.J. Lehotay, K.A. Son, H. Kwon, U. Koesukwiwat, W. Fu, K. Mastovska, E. Hoh and N. Leepipatpiboon, J. Chromatogr. A, 1217, 2548 (2010).

18. R.P.Z. Furlani, K.M. Marcilio, F.M. Leme and S.A.V. Tfouni, Food Chem., 126, 1283 (2011).

19. S.W. Lee, J.-H. Choi, S.-K. Cho, H.-A. Yu, A.M. Abd El-Aty and J.-H. Shim, J. Chromatogr. A, 1218, 4366 (2011).

20. E. Cieslik, A. Sadowska-Rociek, J.M.M. Ruiz and M. Surma-Zadora, Food Chem., 125, 773 (2011). 